\title{
Diffusion with resetting in arbitrary spatial dimension
}

\author{
Martin R. Evans ${ }^{(1)}$ and Satya N. Majumdar ${ }^{(2)}$ \\ (1) SUPA, School of Physics and Astronomy, University of Edinburgh, Mayfield \\ Road, Edinburgh EH9 3JZ, United Kingdom \\ (2) Univ. Paris-Sud, CNRS, LPTMS, UMR 8626, Orsay F-01405, France
}

\begin{abstract}
We consider diffusion in arbitrary spatial dimension $d$ with the addition of a resetting process wherein the diffusive particle stochastically resets to a fixed position at a constant rate $r$. We compute the nonequilibrium stationary state which exhibits non-Gaussian behaviour. We then consider the presence of an absorbing target centred at the origin and compute the survival probability and mean time to absorption of the diffusive particle by the target. The mean absorption time is finite and has a minimum value at an optimal resetting rate $r$. Finally we consider the problem of a finite density of diffusive particles, each resetting to its own initial position. While the typical survival probability of the target at the origin decays exponentially with time regardless of spatial dimension, the average survival probability decays asymptotically as $\exp \left(-A(\ln t)^{d}\right)$ where $A$ is a constant. We explain these findings using an interpretation as a renewal process and arguments invoking extreme value statistics.
\end{abstract}




\section{Introduction}

Diffusion is a fundamental dynamical process dating back to Einstein and the properties of the diffusion equation have been much studied. In this work we consider the problem of diffusion with a stochastic resetting process whereby the diffusive particle is reset to a fixed position (which we usually take to coincide with the particle's initial position) at random times and the diffusive process begins anew. It turns out [1] that resetting fundamentally affects the properties of diffusion.

Resetting holds the system away from any equilibrium state by constantly returning the system to the initial condition. It is thus a simple way of generating a nonequilibrium stationary state. In such states probability currents are non-zero and detailed balance does not hold - for resetting systems a non-vanishing steady-state current is directed towards the resetting position. The nature and properties of nonequilibrium stationary state are questions of fundamental importance within statistical physics [2]. Thus, the resetting paradigm provides a convenient framework within which to study such nonequilibrium properties.

Resetting was first considered in a stochastic multiplicative model of population growth where stochastic resetting events of the population size was shown to lead to a stationary distribution in which the population size has a power-law distribution 3]. A continuous-time random walk model in the presence of a constant drift and resetting has also been studied and the stationary distribution established [4]. Related models of population growth include those involving catastrophic events in which the population is stochastically reduced and reset to some value lower value [5].

The resetting paradigm has a natural realisation in the context of search processes [6,7]. The optimal stochastic search appears as a classic problem in areas as diverse as computer science [8] (e.g. searching for an element in an array) through biochemistry [9] (e.g. a protein searching a binding site) to macrobiology [10 (e.g. a predator seeking its prey). It is also a teasing question in everyday life-how best does one search for lost keys? One general class of search strategies are termed intermittent and combine periods of slow, local motion, termed foraging, in which the target may be detected with periods of fast motion, termed relocation, during which the searcher relocates to new territory (see [7] for a recent review). A diffusive process mimics the foraging phase and a resetting process mimics the relocation. Thus diffusion with stochastic resetting provides a simple realisation of an intermittent search process. Another related search process introduced in [11] is one in which an individual has a random lifetime and when the searcher dies, a new searcher is introduced into the system at the initial starting point. In the mathematical literature, the mean hitting time for a class of random walks in which the walker may choose to restart the walk has been studied recently from an algorithmic point of view $[12$.

In this paper we consider diffusion with stochastic resetting as a fundamental nonequilibrium process in which the statistics of first passage properties may be computed exactly. A simple model of diffusion with stochastic resetting, in which a 
Brownian particle is stochastically reset to its initial position with a constant rate $r$ was defined and studied in [1] with the focus on one spatial dimension. Amongst the results obtained, it was shown in [1] that there exists an optimal resetting rate $r^{*}$ that minimizes the average hitting time to the target. Extensions to space-dependent resetting rate, resetting to a random position with a given distribution and to a spatial distribution of the target were considered in [13]. How the average absorption time is increased when the searcher is only partially absorbed by the target, corresponding to an imperfect searcher, has been studied in [14]. Finally a comparison between the statistics of first passage times for diffusion with stochastic resetting and for an equilibrium dynamics that generates the same stationary state has been made [15].

In the present work we extend these studies to diffusion in arbitrary spatial dimension $d$. In Section 2 we define the model and solve the forward master equation for the probability distribution of the diffusive particle both in the stationary state and relaxing to the stationary state. In Section 3 we compute the survival probability of a fixed absorbing target (or trap) in the presence of a diffusing particle with resetting. We also present a simple interpretation of the result in terms of the extreme value statistics of a renewal process. In Section 4 we compute the optimal mean first absorption time in arbitrary dimension. In Section 5 we consider many diffusive particles in the presence of a single trap and study the average and typical behaviours of the survival probability of the trap. The system furnishes one of the few models for which all these properties can be computed exactly in arbitrary dimensions. Finally we conclude in Section 6 with a summary and outlook.

\section{Diffusive resetting problem in arbitrary spatial dimension}

\subsection{Model definition}

First let us define diffusion with resetting in arbitrary spatial dimension $d$. We consider a single particle (or searcher) in $\mathcal{R}^{d}$ with initial position $\vec{x}_{0}$ at $t=0$ and resetting to position $\vec{X}_{r}$. We stress here that the initial position $\vec{x}_{0}$ and resetting position $\vec{X}_{r}$ are in general distinct, although at the end of some calculations it is convenient to set them to be equal. Our notation for the resetting position thus differs from that of [1].

The position $\vec{x}(t)$ of the particle at time $t$ is updated by the following stochastic

rule [1]: in a small time interval $\mathrm{d} t$ each component $x_{i}$ of the position vector $\vec{x}(t)$ becomes

$$
\begin{aligned}
x_{i}(t+d t) & =\left(X_{r}\right)_{i} \quad \text { with probability } r d t \\
& =x_{i}(t)+\xi_{i}(t) d t \quad \text { with probability }(1-r d t)
\end{aligned}
$$

where $\xi_{i}(t)$ is a Gaussian white noise with mean $\left\langle\xi_{i}(t)\right\rangle=0$ and the two-point correlator $\left\langle\xi_{i}(t) \xi_{j}\left(t^{\prime}\right)\right\rangle=2 D \delta_{i j} \delta\left(t-t^{\prime}\right)$. The dynamics thus consists of a stochastic mixture of resetting to the initial position with rate $r$ (long range move) and ordinary diffusion (local move) with diffusion constant $D$ (see Fig. (1)). 


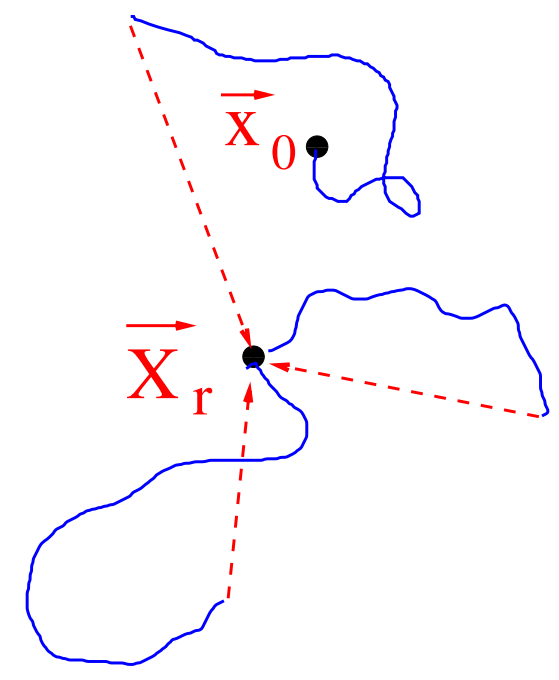

Figure 1. Illustration in $d=2$ of the diffusion with resetting process: the particle starts at initial position $\vec{x}_{0}$ and resets to position $\vec{X}_{r}$ with rate $r$.

\subsection{Solution of Master Equation for diffusion with resetting}

The probability density for the particle to be at position $\vec{x}$ at time $t$, having started from position $\vec{x}_{0}$ at time $t=0$ with resetting to position $\vec{X}_{r}$, should, in principle, be written as $p\left(\vec{x}, t \mid \vec{x}_{0} ; \vec{X}_{r}\right)$. However in the following, in order to lighten the notation, we shall suppress the initial and resetting positions and abbreviate to $p(\vec{x}, t)$.

The forward master equation for the probability density for diffusion with resetting rate $r$ to point $\vec{X}_{r}$ reads

$$
\frac{\partial p(\vec{x}, t)}{\partial t}=D \nabla^{2} p(\vec{x}, t)-r p(\vec{x}, t)+r \delta^{d}\left(\vec{x}-\vec{X}_{r}\right),
$$

with initial condition $p(\vec{x}, 0)=\delta^{d}\left(\vec{x}-\vec{x}_{0}\right)$. The first term on the right hand side (r.h.s) of Equation (2) expresses the diffusive spread of probability; the second term expresses the loss of probability from $\vec{x}$ due to resetting to $\vec{X}_{r}$; the final term corresponds to the gain of probability at $\vec{X}_{r}$ due to resetting from all other positions.

One can write down the solution to 2 in a simple and intuitive way as follows. We first note that the (initial value) diffusive Green function in the absence of resetting $(r=0)$, which we denote $G\left(\vec{x}, t \mid \vec{x}_{0}\right)$, satisfies

$$
\frac{\partial G\left(\vec{x}, t \mid \overrightarrow{x_{0}}\right)}{\partial t}=D \nabla^{2} G\left(\vec{x}, t \mid \vec{x}_{0}\right),
$$

with initial condition $G\left(\vec{x}, t=0 \mid \vec{x}_{0}\right)=\delta^{d}\left(\vec{x}-\vec{x}_{0}\right)$, and is given by

$$
G\left(\vec{x}, t \mid \vec{x}_{0}\right)=\frac{1}{(4 \pi D t)^{d / 2}} \exp \left[-\frac{\left|\vec{x}-\vec{x}_{0}\right|^{2}}{4 D t}\right] \text {. }
$$


Then the probability $p(\vec{x}, t)$ is a sum over two contributions: one which comes from trajectories where no resetting events have occurred in time $t$ and a second contribution which comes from summing over trajectories where the last resetting event occurred at time $t-\tau$. The probability of no resetting events having occurred up to time $t$ is $\mathrm{e}^{-r t}$ and the probability of the last resetting event having occurred at $t-\tau$ (and no resetting events since) is $r \mathrm{e}^{-r \tau}$. Thus the full time-dependent solution can be written down as

$$
p(\vec{x}, t)=\mathrm{e}^{-r t} G\left(\vec{x}, t \mid \vec{x}_{0}\right)+r \int_{0}^{t} \mathrm{~d} \tau \mathrm{e}^{-r \tau} G\left(\vec{x}, \tau \mid \vec{X}_{r}\right) .
$$

The stationary state is attained when $t \gg 1 / r$ where (5) tends to the stationary distribution

$$
p^{*}(\vec{x})=r \int_{0}^{\infty} \mathrm{d} \tau \mathrm{e}^{-r \tau} G\left(\vec{x}, \tau \mid \vec{X}_{r}\right) .
$$

The relaxation to the steady state may be obtained from

$$
p(\vec{x}, t)=p^{*}(\vec{x})+\mathrm{e}^{-r t} G\left(\vec{x}, t \mid \vec{x}_{0}\right)-r \int_{t}^{\infty} \mathrm{d} \tau \mathrm{e}^{-r \tau} G\left(\vec{x}, \tau \mid \vec{X}_{r}\right) .
$$

In order to evaluate the integral in (6) we use the identity (Equation 3.471.9 of [16])

$$
\int_{0}^{\infty} \mathrm{d} t t^{\nu-1} \mathrm{e}^{-\frac{\beta}{t}-\gamma t}=2\left(\frac{\beta}{\gamma}\right)^{\nu / 2} K_{\nu}(2 \sqrt{\beta \gamma})
$$

where $K_{\nu}$ is the modified Bessel function of the second kind (also known as Macdonald function) of order $\nu$. The relevant case of this identity is

$$
\nu=1-d / 2,
$$

and one obtains from (4) and (6), after setting $\vec{x}_{0}=\vec{X}_{r}$

$$
p^{*}(\vec{x})=\left(\frac{\alpha_{0}^{2}}{2 \pi}\right)^{1-\nu}\left(\alpha_{0}\left|\vec{x}-\vec{X}_{r}\right|\right)^{\nu} K_{\nu}\left(\alpha_{0}\left|\vec{x}-\vec{X}_{r}\right|\right),
$$

where

$$
\alpha_{0}=\left(\frac{r}{D}\right)^{1 / 2}
$$

Expression (10), for the stationary distribution of diffusion in the presence of resetting in arbitrary dimension, is the central result of this section. Note that 10 tends to zero as $|\vec{x}| \rightarrow \pm \infty$ and has a cusp at $\vec{x}=\vec{X}_{r}$ (see Figure 2). Also note that 13) is a nonequilibrium stationary state by which it is meant that there is circulation of probability (even in a one-dimensional geometry). This is because resetting implies a source of probability at $\vec{X}_{r}$ while probability is lost from all other values of $\vec{x} \neq \vec{X}_{r}$.

2.2.1. Special Cases $d=1$ and $d=3$ : In the case $d=1(\nu=1 / 2)$ we use the identity

$$
K_{1 / 2}(y)=\left(\frac{\pi}{2 y}\right)^{1 / 2} \mathrm{e}^{-y}
$$

to find that the stationary distribution 10 reduces to

$$
p^{*}(x)=\frac{\alpha_{0}}{2} \exp \left(-\alpha_{0}\left|x-X_{r}\right|\right),
$$




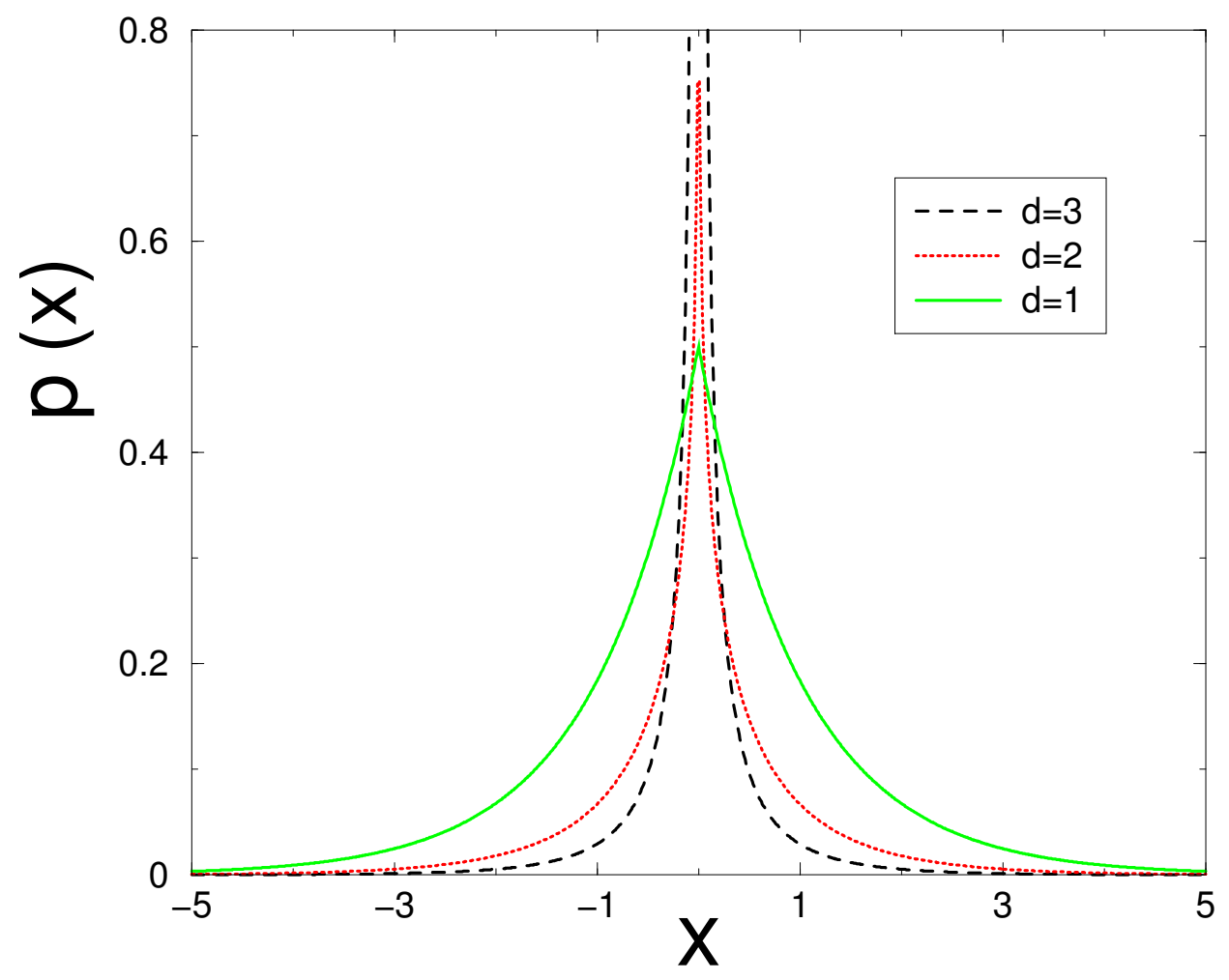

Figure 2. The stationary probability densities $p^{*}(x=|\vec{x}|)$ given by Equation 10 for the case $\alpha_{0}=1$ and $\vec{X}_{r}=0: d=1$ full lines; $d=2$ dotted lines; $d=3$ dashed lines.

thus recovering the result of [1], [13].

Also in the case $d=3(\nu=-1 / 2)$, we may use the identity $K_{-1 / 2}(y)=K_{1 / 2}(y)$ to find a simple form for the stationary distribution

$$
p^{*}(\vec{x})=\frac{\alpha_{0}^{2}}{4 \pi\left|\vec{x}-\vec{X}_{r}\right|} \exp \left(-\alpha_{0}\left|\vec{x}-\vec{X}_{r}\right|\right) .
$$

\section{Survival probability in the presence of a trap at the origin}

\subsection{Trap at origin and length scales of the system}

We now consider the presence of a trap at the origin, i.e., an absorbing $d$-dimensional sphere of radius $a$ centred at $\vec{x}=0$ which absorbs the particle. The particle starts at the initial position $\left|\vec{x}_{0}\right|>a$ and undergoes diffusion with diffusion constant $D$ and stochastic resetting to $\vec{X}_{r}$ with a constant rate $r$. When it reaches the surface of the target sphere, the particle is absorbed (see Figure 3 ).

Once we set $\vec{x}_{0}=\vec{X}_{r}$, there are only three length scales in the system. The parameter $\alpha_{0}$ defined in (11) is an inverse length scale which corresponds to the typical distance diffused by the particle between resets. The other two length scales are $R_{r}=\left|\vec{X}_{r}\right|$, the distance from the origin to resetting position, and $a$ the radius of 

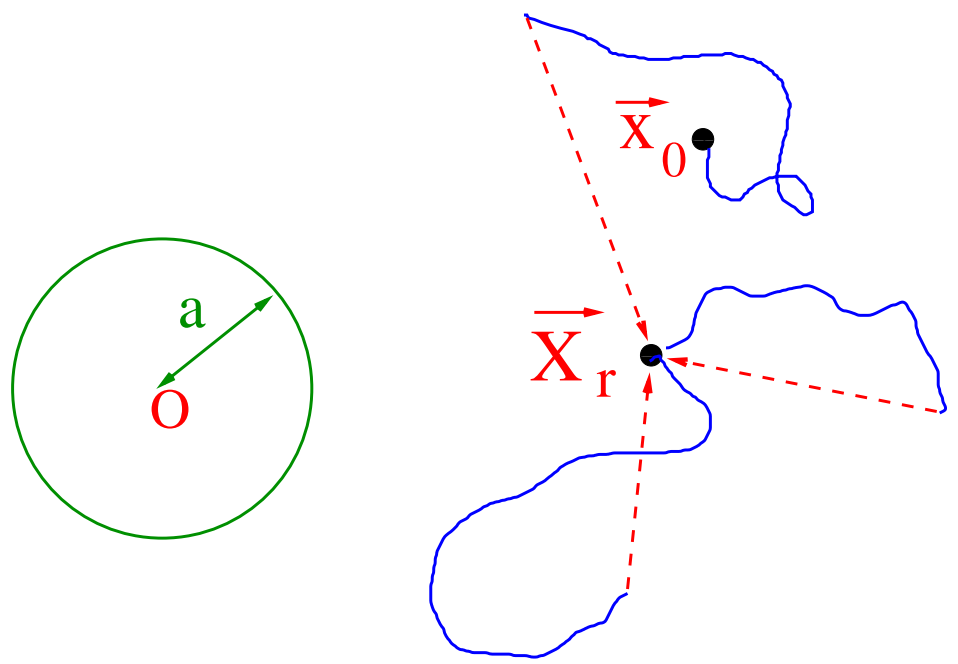

Figure 3. Illustration in $d=2$ of the diffusion of a particle with initial position $\vec{x}_{0}$ and resetting to $\vec{X}_{r}$, in the presence of an absorbing trap of radius $a$ with centre at the origin $O$.

the trap. These three length scales can be combined to define dimensionless reduced variables

$$
\begin{aligned}
\gamma & =\alpha_{0} R_{r} \\
\epsilon & =\frac{a}{R_{r}} .
\end{aligned}
$$

The dimensionless quantity $\gamma$ measures the ratio of the distance $R_{r}$ of the reset point from the target at the origin to the diffusion length $\alpha_{0}^{-1} ; \epsilon$ is simply the ratio of the radius of the absorbing sphere to the distance $R_{r}$ of the reset point to the target at the origin.

\subsection{Computation of survival probability}

To study absorption problems it is most convenient to consider the backward master equation for the survival probability [17]. In this formulation the initial position is considered as a variable. In the presence of resetting it then is important to distinguish the initial position which is a variable (denoted henceforth by $\vec{x}_{0}$ ) from the resetting position $\vec{X}_{r}$ which is fixed. At the end of the calculation we will set $\vec{x}_{0}=\vec{X}_{r}$.

We are interested in the survival probability of a diffusive particle at time $t$, having started at $\vec{x}_{0}$ at $t=0$ with resetting to $\vec{X}_{r}$, which in principle should be denoted $Q\left(\vec{x}_{0}, t ; \vec{X}_{r}\right)$. However, to lighten the notation we will suppress the resetting position and write $Q\left(\vec{x}_{0}, t\right)$. In the same way we will write its Laplace transform, defined below (20), as $q\left(\vec{x}_{0}, s\right)$. 
The backward master equation for $Q\left(\vec{x}_{0}, t\right)$ is constructed by considering events in the first infinitesimal time interval $\mathrm{d} t$ : the components $\left(x_{0}\right)_{i}$ of the initial position become

$$
\begin{aligned}
\left(x_{0}\right)_{i} & \rightarrow\left(X_{r}\right)_{i} \quad \text { with probability } r \mathrm{~d} t \\
& \rightarrow\left(x_{0}\right)_{i}+\xi_{i}(0) \mathrm{d} t \quad \text { with probability } 1-r \mathrm{~d} t
\end{aligned}
$$

where $\xi_{i}(0)$ is the $i$ th component of the initial noise. Then

$$
Q\left(\vec{x}_{0}, t+\mathrm{d} t\right)=(1-r \mathrm{~d} t)\left\langle Q\left(\vec{x}_{0}+\vec{\eta}(0) \mathrm{d} t, t\right)\right\rangle+r \mathrm{~d} t Q\left(\vec{X}_{r}, t\right),
$$

where $\langle\cdot\rangle$ indicates averaging over the initial noise. Performing the average and taking the $\mathrm{d} t \rightarrow 0$ limit yields, for $\left|\vec{x}_{0}\right|>a$,

$$
\frac{\partial Q\left(\vec{x}_{0}, t\right)}{\partial t}=D \nabla_{\vec{x}_{0}}^{2} Q\left(\vec{x}_{0}, t\right)-r Q\left(\vec{x}_{0}, t\right)+r Q\left(\vec{X}_{r}, t\right)
$$

with boundary and initial conditions $Q\left(\left|\vec{x}_{0}\right|=a, t\right)=0$ and $Q\left(\vec{x}_{0}, 0\right)=1$ for $\left|\vec{x}_{0}\right|>a$.

The Laplace transform

$$
q\left(\vec{x}_{0}, s\right)=\int_{0}^{\infty} \mathrm{d} t \mathrm{e}^{-s t} Q\left(\vec{x}_{0}, t\right)
$$

satisfies

$$
D \nabla_{\vec{x}_{0}}^{2} q\left(\overrightarrow{x_{0}}, s\right)-(r+s) q\left(\vec{x}_{0}, s\right)=-1-r q\left(\vec{X}_{r}, s\right) .
$$

The solution to the homogeneous equation

$$
D \nabla_{\vec{x}_{0}}^{2} q\left(\vec{x}_{0}, s\right)-(r+s) q\left(\vec{x}_{0}, s\right)=0,
$$

which is radially symmetric about the origin and does not diverge as $\left|\vec{x}_{0}\right| \rightarrow \infty$, is

$$
q_{\mathrm{hom}}\left(\vec{x}_{0}, s\right)=\left(\alpha\left|\vec{x}_{0}\right|\right)^{\nu} K_{\nu}\left(\alpha\left|\vec{x}_{0}\right|\right)
$$

where now

$$
\alpha(s)=\left(\frac{r+s}{D}\right)^{1 / 2}
$$

and as before, $\nu=1-d / 2$. Note that $\alpha(0)=\alpha_{0}$ given in (11). Hereafter, we write $\alpha \equiv \alpha(s)$ for brevity.

In order to determine the solution to (21) we write

$$
q\left(\overrightarrow{x_{0}}, s\right)=B\left|\vec{x}_{0}\right|^{\nu} K_{\nu}\left(\alpha\left|\vec{x}_{0}\right|\right)+C
$$

where $C$ is independent of $\left|\vec{x}_{0}\right|$. Then the absorbing boundary condition implies that $C=-B a^{\nu} K_{\nu}(\alpha a)$ and substituting (25) back into 21) determines

$$
B=-\frac{1}{s a^{\nu} K_{\nu}(\alpha a)+r\left|\vec{X}_{r}\right|^{\nu} K_{\nu}\left(\alpha\left|\vec{X}_{r}\right|\right)}
$$

Therefore

$$
q\left(\vec{x}_{0}, s\right)=\frac{a^{\nu} K_{\nu}(\alpha a)-\left|\vec{x}_{0}\right|^{\nu} K_{\nu}\left(\alpha\left|\vec{x}_{0}\right|\right)}{r\left|\vec{X}_{r}\right|^{\nu} K_{\nu}\left(\alpha\left|\vec{X}_{r}\right|\right)+s a^{\nu} K_{\nu}(\alpha a)}
$$


In particular, setting the starting position $\vec{x}_{0}$ equal to the resetting position $\vec{X}_{r}$, and using isotropy, we have

$$
q\left(\vec{X}_{r}, s\right)=q\left(R_{r}, s\right)=\frac{a^{\nu} K_{\nu}(\alpha a)-R_{r}^{\nu} K_{\nu}\left(\alpha R_{r}\right)}{r R_{r}^{\nu} K_{\nu}\left(\alpha R_{r}\right)+s a^{\nu} K_{\nu}(\alpha a)},
$$

where $R_{r}=\left|\vec{X}_{r}\right|$. Equation 27) is the main result of this section and is an exact expression for the Laplace transform of the survival probability in arbitrary dimension $\nu=1-d / 2$.

Having obtained this expression one would ideally wish to invert the Laplace transform in order to obtain

$$
Q\left(R_{r}, t\right) \equiv Q\left(\left|\vec{x}_{0}\right|=R_{r}, t ;\left|\vec{X}_{r}\right|=R_{r}\right) .
$$

However, it appears difficult to invert the Laplace transform (27) explicitly for all parameters. Nevertheless there are two limits where one may extract the forms of $Q\left(R_{r}, t\right)$ : (i) the natural scaling regime $r \rightarrow 0, t \rightarrow \infty$ with scaling variable $y=r t$ fixed; (ii) for fixed $r$, the large $t$ limit.

\subsection{Scaling regime}

In the scaling regime, we consider the limit $r \rightarrow 0, s \rightarrow 0$ where

$$
\lambda=\frac{s}{r} .
$$

The scaling variable is

$$
y=r t \text {. }
$$

In this limit we obtain the scaling distribution

$$
Q\left(R_{r}, t\right) \rightarrow F\left(R_{r}, y\right)
$$

and (27) becomes

$$
q\left(R_{r}, s\right) \rightarrow f\left(R_{r}, \lambda\right)=\int_{0}^{\infty} \mathrm{d} y \mathrm{e}^{-\lambda y} \frac{F\left(R_{r}, y\right)}{r}
$$

i.e. we obtain a simple scaling form of the Laplace transform $f\left(R_{r}, \lambda\right)$. We may then invert the Laplace transform in 32 with respect to $\lambda$ to obtain the scaling form $F\left(R_{r}, y\right)$ for the survival probability.

To proceed, we use the small argument expansion

$$
\begin{aligned}
K_{\nu}(x) & \simeq-\ln (x / 2)-\gamma_{E} \quad \text { if } \quad \nu=0 \\
& \simeq \frac{\Gamma(\nu)}{2}\left(\frac{2}{x}\right)^{\nu}+\frac{\Gamma(-\nu)}{2}\left(\frac{2}{x}\right)^{-\nu}+\ldots \quad \text { if } \quad 0<\nu<1, \\
& \simeq \frac{\Gamma(\nu)}{2}\left(\frac{2}{x}\right)^{\nu}-\frac{\Gamma(\nu-1)}{2}\left(\frac{2}{x}\right)^{\nu-2}+\ldots \quad \text { if } \quad \nu>1,
\end{aligned}
$$

where $\gamma_{E}=0.5772 \ldots$ is the Euler constant. 
We consider separately the different cases $\nu>0, \nu=0, \nu<0$ corresponding to $d<2, d=2, d>2$ respectively (using $\nu=1-d / 2$ ).

i) $1>\nu>0(0<d<2)$ : In this case both terms in $(34)$ are required and one finds

$$
q\left(R_{r}, s\right) \simeq \frac{\Gamma(-\nu)}{\Gamma(\nu)} \frac{r^{\nu-1}(1+\lambda)^{\nu-1}}{(4 D)^{\nu}}\left[a^{2 \nu}-R_{r}^{2 \nu}\right] .
$$

One may then invert (36) using 32 to obtain the final result in the scaling limit

$$
Q\left(R_{r}, t\right)=F\left(R_{r}, r t\right)
$$

where the scaling function is given by

$$
F\left(R_{r}, y\right)=\frac{1}{\nu \Gamma(\nu)}\left(\frac{\gamma}{2}\right)^{2 \nu}\left(1-\epsilon^{2 \nu}\right) \mathrm{e}^{-y} y^{-\nu}
$$

and, as usual, $\gamma=R_{r}(r / D)^{1 / 2}$ and $\epsilon=a / R_{r}$.

In particular, the $d=1(\nu=1 / 2)$ case is

$$
F\left(R_{r}, y\right)=\frac{\gamma}{\sqrt{\pi}} \frac{\mathrm{e}^{-y}}{y^{1 / 2}} .
$$

Note that in the limit $y \rightarrow 0$

$$
F\left(R_{r}, y\right) \simeq \frac{R_{r}}{\sqrt{D \pi}}\left(\frac{r}{y}\right)^{1 / 2}=\frac{R_{r}}{\sqrt{D \pi t}},
$$

which recovers the usual $1 d$ diffusive survival probability at large $t$. Also note that for $y$ large the survival probability decays exponentially.

ii) $\nu=0(d=2)$ : In the case $d=2$, which corresponds to $\nu=0$, the leading order expansion of (27) in the limit $r \rightarrow 0, s \rightarrow 0$ is

$$
q\left(R_{r}, s\right) \simeq \frac{2 \ln \epsilon}{(s+r) \ln r} .
$$

Inverting the Laplace transform yields

$$
Q\left(R_{r}, t\right) \simeq \frac{2 \ln \epsilon}{\ln r} \mathrm{e}^{-r t}
$$

So in the case $d=2$ a scaling form, which is solely a function of $y$ and $\epsilon$, does not emerge.

iii) $\nu<0(d>2)$. In the case $d>2$, which corresponds to $\nu<0$, we can use the symmetry $K_{-\nu}(x)=K_{\nu}(x)$. Then it turns out that only the leading order term in the small $x$ expansions 34 35 matters. Substituting this in (27) gives in the scaling limit

$$
q\left(R_{r}, s\right) \simeq \frac{1}{r} \frac{1-\epsilon^{-2 \nu}}{\left(\lambda+\epsilon^{-2 \nu}\right)} .
$$

Inverting the Laplace transform yields

$$
F\left(R_{r}, y\right)=\left(1-\epsilon^{-2 \nu}\right) \mathrm{e}^{-y \epsilon^{-2 \nu}} .
$$

Thus for $d>2$ the scaling form decays exponentially in the scaling variable $y$. 


\subsection{Long-time asymptotics of Survival Probability}

The long-time asymptotics of the survival probability for fixed $r$ may be deduced from the inversion of 27

$$
Q\left(R_{r}, t\right)=\int_{C} \frac{\mathrm{d} s \mathrm{e}^{s t}}{2 \pi i} \frac{a^{\nu} K_{\nu}(\alpha a)-R_{r}^{\nu} K_{\nu}\left(\alpha R_{r}\right)}{r R_{r}^{\nu} K_{\nu}\left(\alpha R_{r}\right)+s a^{\nu} K_{\nu}(\alpha a)},
$$

where $C$ is the Bromwich contour to the right of any singularities in the complex $s$ plane. We may rewrite (45) as

$$
Q\left(R_{r}, t\right)=\int_{C} \frac{\mathrm{d} s}{2 \pi i} \frac{\mathrm{e}^{s t}}{r}\left[\frac{r+g(s)}{s-g(s)}\right]
$$

where

$$
g(s)=-\frac{r}{\epsilon^{\nu}} \frac{K_{\nu}\left(\alpha(s) R_{r}\right)}{K_{\nu}(\alpha(s) a)}
$$

with $\epsilon=a / R_{r}$ and $\alpha(s)=\sqrt{\frac{r+s}{D}}$. The analytic structure in the complex $s$ plane of the integrand of 46 is a branch point $s=-r$ coming from $\alpha(s)$, and a simple pole at $s=s_{0}$ which satisfies

$$
s_{0}=g\left(s_{0}\right) .
$$

In the long-time limit the dominant contribution to 46 will come from the pole at $s_{0}$ which yields by the residue theorem

$$
Q\left(R_{r}, t\right) \simeq \frac{\mathrm{e}^{s_{0} t}}{r} \frac{\left[r+s_{0}\right]}{1-g^{\prime}\left(s_{0}\right)}
$$

Making the substitution

$$
s_{0}=r\left(u_{0}-1\right) \quad 0<u_{0}<1,
$$

equation (48) becomes a transcendental equation for $u_{0}$

$$
u_{0}=1-\frac{1}{\epsilon^{\nu}} \frac{K_{\nu}\left(\gamma u_{0}^{1 / 2}\right)}{K_{\nu}\left(\gamma \epsilon u_{0}^{1 / 2}\right)},
$$

where $\gamma=\alpha_{0} R_{r}$. Thus the asymptotic behaviour of the survival probability is

$$
\ln Q\left(R_{r}, t\right) \sim r\left(u_{0}-1\right) t
$$

where $u_{0}$ satisfies 51 .

In the limit $\gamma \gg 1$ (and $\gamma \epsilon \gg 1$ ) we can use the asymptotic behaviour

$$
K_{\nu}(y) \simeq\left(\frac{\pi}{2 y}\right)^{1 / 2} \mathrm{e}^{-y}[1+O(1 / y)] \text { for large } y
$$

to obtain the behaviour of $u_{0}$

$$
u_{0} \simeq 1-\epsilon^{1 / 2-\nu} \mathrm{e}^{-\gamma(1-\epsilon)} .
$$

Then (49) takes the form

$$
Q\left(R_{r}, t\right) \sim \exp \left(-r t \epsilon^{1 / 2-\nu} \mathrm{e}^{-\gamma(1-\epsilon)}\right) .
$$

This expression has the form of a Gumbel distribution, the origin of which we shall now discuss. 
3.4.1. Renewal process and extreme value statistics interpretation In order to better understand the result (55) we consider the diffusion with resetting process as a process which is renewed each time the particle is reset - the renewed process has no memory of its past history. We shall consider the long time regime where the number of resets to position $\vec{X}_{r}$ in time $t$ is the mean number of resets $N=r t$ plus fluctuations of $O\left(t^{1 / 2}\right)$.

In order for the target to survive up to time $t$ it must survive through each of the resets. Then we can write the asymptotic behaviour as

$$
Q\left(R_{r}, t\right) \sim\left(\left\langle P_{S}^{\text {diff }}(\tau)\right\rangle_{\tau}\right)^{r t}
$$

where the $P_{S}^{\text {diff }}$ is the survival probability of a diffusive particle up to time $\tau$ and $\langle\cdot\rangle_{\tau}$ denotes an average over the duration of the reset (time until next resetting event). As resetting is a Poisson process we have

$$
\left\langle P_{S}^{\text {diff }}(\tau)\right\rangle_{\tau}=\int_{0}^{\infty} \mathrm{d} \tau r e^{-r \tau} P_{S}^{\text {diff }}(\tau)=r q^{\text {diff }}\left(R_{r}, r\right)
$$

where now $q^{\text {diff }}\left(R_{r}, r\right)$ is the Laplace transform, with Laplace variable $s$ replaced by $r$, of the survival probability of a diffusive particle with an absorbing target at the origin. The expression for $q^{\text {diff }}\left(R_{r}, r\right)$ is easy to construct using, for example, a backward master equation approach and is well-known in the literature (see e.g. [18]):

$$
\begin{aligned}
q^{\mathrm{diff}}\left(R_{r}, r\right) & =\frac{1}{r}\left[1-\left(\frac{R_{r}}{a}\right)^{\nu} \frac{K_{\nu}\left(R_{r}(r / D)^{1 / 2}\right)}{K_{\nu}\left(a(r / D)^{1 / 2}\right)}\right] \\
& =\frac{1}{r}\left[1-\epsilon^{-\nu} \frac{K_{\nu}(\gamma)}{K_{\nu}(\gamma \epsilon)}\right] .
\end{aligned}
$$

We then have

$$
\begin{aligned}
Q\left(R_{r}, t\right) & \sim \exp \left\{r t \ln \left[1-\epsilon^{-\nu} \frac{K_{\nu}(\gamma)}{K_{\nu}(\gamma \epsilon)}\right]\right\} \\
& \simeq \exp \left[-r t \epsilon^{1 / 2-\nu} \mathrm{e}^{-\gamma(1-\epsilon)}\right],
\end{aligned}
$$

where we have used the asymptotic behaviour (53) for large $\gamma$ and $\epsilon \gamma$, thus recovering (55).

One may relate this result to the Gumbel distribution for the extremum of independent random variables [19] as follows. As we have seen above, for the target to survive it must survive through $N$ resets. Thus the leftmost point the searcher has reached in the $N$ resets must be less than $R_{r}$, the distance to the target. In other words, the maximum of $N$ independent random variables must be less than $R_{r}$. Thus the Gumbel distribution, which is the scaling form for the cumulative distribution of the maximum of $N$ independent random variables with distribution decaying faster than a power law, naturally emerges.

\subsection{Generalisation to a resetting distribution}

The computation of the survival probability can easily be generalised to the case of a resetting distribution $\mathcal{P}(\vec{z})[13]$ where $\vec{z}$ is a randomly chosen resetting position. 
Equation (1) is modified to

$$
\begin{aligned}
x_{i}(t+d t) & =z_{i} \quad \text { with probability } r \mathcal{P}(\vec{z}) d t \\
& =x_{i}(t)+\xi_{i}(t) d t \quad \text { with probability }(1-r d t) .
\end{aligned}
$$

Also, if we choose the distance of the initial position from the origin, $R=\left|\vec{x}_{0}\right|$ to be drawn from the same distribution $\mathcal{P}(R)$ we find that the Laplace transform of the survival probability averaged over the resetting distribution becomes

$$
\bar{q}(s)=\frac{a^{\nu} K_{\nu}(\alpha a)-\int \mathrm{d} R R^{\nu} K_{\nu}(\alpha R) \mathcal{P}(R)}{s a^{\nu} K_{\nu}(\alpha a)+r \int \mathrm{d} R R^{\nu} K_{\nu}(\alpha R) \mathcal{P}(R)} .
$$

Equation (63) gives the survival probability for a diffusive particle with a trap at the origin for abitrary resetting distribution, thus generalising the result (27) for a fixed

resetting position. Equation (63) may be used to study the optimal choices of resetting distribution to optimize for example the mean time to absorption [13.

\section{Mean First-passage time to absorption by sphere of radius $a$ in arbitrary dimensions}

We now consider the mean time to absorption in the presence of an absorbing trap in the case of diffusion with resetting to point $\vec{X}_{r}$.

\subsection{Mean time to absorption}

The mean time to absorption by the trap at the origin, with coincident initial and resetting positions, can be computed from the survival probability $Q\left(R_{r}, t\right)$

$$
\begin{aligned}
T\left(R_{r}\right) & =-\int_{0}^{\infty} \mathrm{d} t t \frac{\partial Q\left(R_{r}, t\right)}{\partial t} \\
& =q\left(R_{r}, s=0\right) .
\end{aligned}
$$

(The integration by parts requires $Q\left(R_{r}, t\right)$ to vanish faster than $1 / t$ for large $t$.) Thus, the mean time to absorption is obtained by setting $s=0$ in 27

$$
T\left(R_{r}\right)=\frac{1}{r}\left[\left(\frac{a}{R_{r}}\right)^{\nu} \frac{K_{\nu}\left(\alpha_{0} a\right)}{K_{\nu}\left(\alpha_{0} R_{r}\right)}-1\right] .
$$

Note that for $d \leq 2$, (65) has a finite limit as $a \rightarrow 0$, but for $d>2$, (65) diverges as $a \rightarrow 0$.

Let us recall that

$$
K_{\nu}(z)=K_{-\nu}(z) .
$$

Then using the dimensionless quantities (15), (16) one may rewrite 65 as

$$
T=\frac{1}{\gamma^{2}}\left[\epsilon^{\nu} \frac{K_{|\nu|}(\epsilon \gamma)}{K_{|\nu|}(\gamma)}-1\right] \frac{R_{r}^{2}}{D},
$$

where, as usual, $\gamma=\alpha_{0} R_{r}=\sqrt{r / D} R_{r}$ and we have suppressed the $R_{r}$ dependence of $T\left(R_{r}\right)$ for convenience. The first point to note is that $T$ is finite for $0<r<\infty$. It 
diverges as $r \rightarrow 0$ as $T \sim r^{-1 / 2}$ in $d=1,3$ and as $T \sim 1 /(r|\ln r|)$ in $d=2$ as can be checked using the small argument expansion of the modified Bessel functions 33 35). It also diverges exponentially in $r$ as $r \rightarrow \infty$ which can be easily checked using the asymptotic behaviour (53). The $r \rightarrow 0$ limit recovers the well-known result that the mean time for a diffusive particle to reach the origin diverges. The $r \rightarrow \infty$ limit merely expresses the fact that as the reset rate increase the diffusing particle has less time between resets to reach the origin.

Now, for fixed $\epsilon$ and fixed $R_{r}^{2} / D$, we wish to see how $T$ in Eq. (67) behaves as a function of the reduced variable $\gamma=\sqrt{r / D} R_{r}$. Since $T$ diverges in the limits $\gamma \rightarrow 0$ and $\gamma \rightarrow \infty$, there is a unique minimum of $T$ at the optimal value $\gamma^{*}$ where $\mathrm{d} T / \mathrm{d} \gamma=0$. This optimal $\gamma^{*}$ is evidently a complicated function of $\epsilon$ and in general, is hard to determine explicitly. However, we are interested in the limit $\epsilon=a / R_{r} \rightarrow 0$ limit such that the target size is small compared to the particles initial position. In this limit, one can obtain explicit results as we now show.

\subsection{Small $\epsilon$ limit}

Taking $\epsilon \rightarrow 0$ limit in Eq. (67), upon using the asymptotic behaviour of $K_{\nu}(z)$ in Eqs. (33) and (34), gives for $\nu=1-d / 2 \neq 0$

$$
T \rightarrow \frac{1}{\gamma^{2}}\left[\epsilon^{\nu-|\nu|} \Gamma(|\nu|) 2^{|\nu|-1} \frac{\gamma^{-|\nu|}}{K_{|\nu|}(\gamma)}-1\right] \frac{R_{r}^{2}}{D} .
$$

4.2.1. Case $d<2$ : Consider first the case $d<2$. In this case, $\nu=1-d / 2$ and $|\nu|=1-d / 2$ and hence the $\epsilon$ dependence of $T$ in Eq. (68) just drops out in the $\epsilon \rightarrow 0$ limit. This is consistent with the fact that for $d<2$, one does not need to have a finite size of the target and there is well defined point target limit. Hence, for $d<2$, we find the exact result

$$
T_{d<2}=\frac{1}{\gamma^{2}}\left[2^{-d / 2} \Gamma\left(1-\frac{d}{2}\right) \frac{\gamma^{d / 2-1}}{K_{1-\frac{d}{2}}(\gamma)}-1\right] \frac{R_{r}^{2}}{D} .
$$

In particular, for $d=1$, using $(12)$ we recover the 1 -d result [1]

$$
T_{d=1}=\frac{1}{\gamma^{2}}\left[\mathrm{e}^{\gamma}-1\right] \frac{R_{r}^{2}}{D}
$$

which has a unique minimum at $\gamma^{*}=\gamma_{1}=1.59362 \ldots$ where $\gamma_{1}$ is the unique positive root of $\gamma_{1}-2\left(1-e^{-\gamma_{1}}\right)=0$. For general $d<2$, one can similarly minimize Eq. 69 and obtain the optimal $\gamma^{*}$.

4.2.2. Case $d>2$ : In the opposite case $d>2$, the $\epsilon$ dependence of $T$ does not drop out of Eq. (68), indicating that for $d>2$, one needs a finite target size as otherwise a point particle will never meet a point target. In this case, to leading order in $\epsilon$, Eq. (68) gives

$$
T_{d>2} \rightarrow\left[\frac{\gamma^{-1-d / 2}}{K_{\frac{d}{2}-1}(\gamma)}\right] \Gamma\left(\frac{d}{2}-1\right) 2^{d / 2-2} \epsilon^{2-d} \frac{R_{r}^{2}}{D}
$$


One can then minimize $T$ as a function of $\gamma$. The optimal value $\gamma^{*}$ is obtained by setting $\frac{\mathrm{d} T}{\mathrm{~d} \gamma}=0$ and $\gamma=\gamma^{*}$. Taking the derivative of Eq. 71 with respect to $\gamma$ and using the property of the modified Bessel function [16],

$$
y K_{\nu}^{\prime}(y)+\nu K_{\nu}(y)=-y K_{\nu-1}(y)
$$

we obtain $\gamma^{*}$ as a root of the equation

$$
2 K_{\frac{d}{2}-1}\left(\gamma^{*}\right)-\gamma^{*} K_{\frac{d}{2}-2}\left(\gamma^{*}\right)=0 .
$$

A particular simplification occurs for $d=3$ where one can use $K_{-1 / 2}(y)=K_{1 / 2}(y)$ to obtain explicitly $\gamma^{*}=2$. Hence, using $K_{1 / 2}(y)=\sqrt{\pi / 2 y} e^{-y}$, the optimal mean first-passage time for $d=3$ is given explicitly by

$$
T_{d=3}^{*}=\mathrm{e}^{2} \frac{R_{r}^{2}}{D} \frac{1}{\epsilon} .
$$

In the marginal dimension $d=2$, one has $\nu=0$ and hence from Eq. 67) we obtain

$$
T_{d=2}=\frac{1}{\gamma^{2}}\left[\frac{K_{0}(\epsilon \gamma)}{K_{0}(\gamma)}-1\right] \frac{R_{r}^{2}}{D} .
$$

Taking again the $\epsilon \rightarrow 0$ limit using Eq. (33) gives, to leading order in small $\epsilon$,

$$
T_{d=2} \rightarrow \frac{-\ln (\epsilon)}{\gamma^{2} K_{0}(\gamma)} \frac{R_{r}^{2}}{D} .
$$

Once again, one can minimize $T$ as a function of $\gamma$. The optimal $\gamma^{*}$ is obtained by solving $\frac{\mathrm{d} T}{\mathrm{~d} \gamma}=0$ and is given by the root of the equation

$$
2 K_{0}\left(\gamma^{*}\right)-\gamma^{*} K_{1}\left(\gamma^{*}\right)=0
$$

which gives $\gamma^{*}(d=2)=1.55265 \ldots$. Substituting this result in Eq. (76), we find that the exact optimal mean first-passage time in $d=2$

$$
T_{d=2}^{*} \rightarrow 2.07679 \ldots[-\ln (\epsilon)] \frac{R_{r}^{2}}{D} .
$$

\section{Many Searchers}

\subsection{Average and typical behaviour: annealed and quenched averages}

We now consider the problem of many independent searchers (diffusive particles) and the survival probability of a stationary target at the origin defined as in Section 4.1. Specifically, we consider $N$ diffusive particles labelled $\mu=1, \ldots, N$, each of which is reset to its own resetting position $\vec{X}_{\mu}$ with rate $r$. We also take the initial position of each searcher to be identical to its resetting position.

The survival probability of the target is given by

$$
P_{s}(t)=\prod_{\mu=1}^{N} Q\left(\vec{X}_{\mu}, t\right)
$$


where $Q\left(\vec{X}_{\mu}, t\right)$ is the survival probability in the single searcher problem considered in Section 3. Note that in the absence of resetting $(r=0)$ this survival probability $P_{s}(t)$ has been studied in all dimensions 20 23.

We consider the $N$ resetting positions to be distributed uniformly with density $\rho$ outside of the target volume and consequently, $P_{s}(t)$ is a random variable. Its average is simply $P_{s}^{\text {av }}(t)=\left\langle P_{s}(t)\right\rangle_{\vec{x}}$ where $\langle\cdot\rangle_{\vec{X}}$ denotes averages over $\vec{X}_{\mu}$ 's. However, as we shall see, for a typical resetting configuration $P_{s}(t)$ is not captured by the average. This is because the average may be dominated by rare distributions of the resetting positions of the searchers for which the survival probability is much larger than is typical. One can think of the resetting positions as initial conditions which are remembered for all time by the system through the resetting dynamics.

The typical $P_{s}(t)$ can be extracted by first averaging over the logarithm of $P_{s}(t)$ followed by exponentiating: $P_{s}^{\text {typ }}(t)=\exp \left[\left\langle\ln P_{s}(t)\right\rangle_{\vec{X}}\right]$. One can draw an analogy to a disordered system with $P_{s}(t)$ playing the role of partition function $Z$ and $X_{\mu}$ 's corresponding to disorder variables. Thus the average and typical behaviour correspond respectively to the annealed average (where one averages the partition function $Z$ ) and the quenched average (where one averages the free energy $\ln Z$ ) in disordered systems.

To compute the average behaviour of (79) (the annealed case) we may write

$$
\begin{aligned}
P_{s}^{\mathrm{av}}(t) & =\left[\langle Q(\vec{X}, t)\rangle_{\vec{X}}\right]^{N} \\
& =\exp \left\{N \ln \left[1-\langle 1-Q(\vec{X}, t)\rangle_{\vec{X}}\right]\right\}
\end{aligned}
$$

where $\langle\cdot\rangle_{\vec{X}}$ denotes an average over the resetting position $\vec{X}$. We begin by considering $\vec{X}$ to be distributed uniformly over a volume $V$ comprising a sphere of radius $L$ with the target volume, which is a sphere of radius $a$, removed. Noting that $Q(\vec{X}, t)=Q(R, t)$, where $R=|\vec{X}|$, we obtain

$$
\langle 1-Q(\vec{X}, t)\rangle_{\vec{X}}=1-\frac{1}{V} \int_{R>a} \mathrm{~d} R \Gamma_{d} R^{d-1}(1-Q(R, t)),
$$

where

$$
\Gamma_{d}=\frac{2 \pi^{d / 2}}{\Gamma(d / 2)}
$$

is the surface area of a $d$-dimensional unit sphere. Letting $N, L \rightarrow \infty$ but keeping the density of walkers $\rho=N / V$ fixed, we obtain

$$
P_{s}^{\mathrm{av}}(t) \rightarrow \exp \left[-\rho \Gamma_{d} \int_{a}^{\infty} \mathrm{d} R R^{d-1}(1-Q(R, t))\right] \equiv \exp \left[-\rho I_{1}(t)\right] .
$$

On the other hand, the typical behaviour (the quenched case) $P_{s}^{\text {typ }}(t)=$ $\exp \left[\left\langle\ln \left(P_{s}(t)\right)\right\rangle_{X}\right]$ can be expressed as

$$
P_{s}^{\text {typ }}(t)=\exp \left\{\sum_{\mu=1}^{N}\left\langle\ln \left[Q\left(\vec{X}_{\mu}, t\right)\right]\right\rangle_{\vec{X}_{\mu}}\right\}=\exp \left\{N\langle\ln [Q(\vec{X}, t)]\rangle_{\vec{X}}\right\}
$$

where

$$
\langle\ln [Q(\vec{X}, t)]\rangle_{\vec{X}}=\frac{1}{V} \int_{R>a} \mathrm{~d} R \Gamma_{d} R^{d-1} \ln [Q(R, t)]
$$


In the limit $N, L \rightarrow \infty$ with density of walkers $\rho=N / V$ fixed, we obtain

$$
P_{s}^{\text {typ }}(t)=\exp \left[\rho \Gamma_{d} \int_{a}^{\infty} \mathrm{d} R R^{d-1} \ln Q(R, t)\right] \equiv \exp \left[-\rho I_{2}(t)\right] .
$$

Thus the determination of the average and typical behaviour reduces to the evaluation of two integrals:

$$
\begin{aligned}
& I_{1}=\Gamma_{d} \int_{a}^{\infty} \mathrm{d} R R^{d-1}[1-Q(R, t)] \\
& I_{2}=-\Gamma_{d} \int_{a}^{\infty} \mathrm{d} R R^{d-1} \ln [Q(R, t)] .
\end{aligned}
$$

\subsection{Average behaviour}

The Laplace transform of $88 \tilde{I}_{1}(s)=\int_{0}^{\infty} I_{1}(t) e^{-s t} \mathrm{~d} t$ can be determined using (27):

$$
\begin{aligned}
\tilde{I}_{1}(s) & =\Gamma_{d} \int_{a}^{\infty} \mathrm{d} R R^{d-1}\left[\frac{1}{s}-q(R, s)\right] \\
& =\Gamma_{d} \frac{(r+s)}{s} \int_{a}^{\infty} \mathrm{d} R R^{d-1}\left[r+s\left(\frac{a}{R}\right)^{\nu} \frac{K_{\nu}(\alpha a)}{K_{\nu}(\alpha R)}\right]^{-1} .
\end{aligned}
$$

We now wish to determine the small $s$ behaviour. First note that for large $x$

$$
K_{\nu}(x) \sim\left(\frac{\pi}{2 x}\right)^{1 / 2} \mathrm{e}^{-x}
$$

Therefore in the integral (90) the term $r$ in the square bracket will dominate upto some length scale $R^{*}$ and we can determine the leading behaviour as

$$
\begin{aligned}
\tilde{I}_{1}(s) & \simeq \Gamma_{d} \frac{(r+s)}{r s} \int_{a}^{R^{*}} \mathrm{~d} R R^{d-1} \\
& \simeq \Gamma_{d} \frac{(r+s)}{r s} \frac{\left(R^{*}\right)^{d}}{d},
\end{aligned}
$$

where we have assumed $R^{*} \gg a$.

In order to deduce $R^{*}$ we note that it is defined by

$$
\left(\frac{a}{R_{r}}\right)^{\nu} \frac{K_{\nu}(\alpha a)}{K_{\nu}\left(\alpha R_{r}\right)} \simeq \frac{r}{s}
$$

Using the asymptotic behaviour 91 of $K_{\nu}\left(\alpha R^{*}\right)$ for $R^{*}$ large we have

$$
\frac{\mathrm{e}^{\alpha_{0} R^{*}}}{\left(R^{*}\right)^{\nu-1 / 2}} \simeq\left(\frac{\pi}{2 \alpha_{0}}\right)^{1 / 2} \frac{1}{a^{\nu} K_{\nu}\left(\alpha_{0} a\right)} \frac{r}{s},
$$

therefore as $s \rightarrow 0$ we obtain

$$
R^{*} \simeq \frac{1}{\alpha_{0}} \ln \left(\frac{r}{s}\right)
$$

Hence,

$$
\tilde{I}_{1}(s) \simeq \frac{\Gamma_{d}}{d s} \frac{1}{\alpha_{0}^{d}}\left[\ln \left(\frac{r}{s}\right)\right]^{d} .
$$

Inverting the Laplace transform one then obtains the large time asymptotic behaviour of $I_{1}(t)$

$$
I_{1}(t) \simeq \frac{\Gamma_{d}}{d \alpha_{0}^{D}}[\ln t]^{d}
$$


and leading large time behaviour

$$
P_{s}^{\mathrm{av}}(t) \sim \exp \left[-\rho \frac{\Gamma_{d}}{d \alpha_{0}^{d}}(\ln t)^{d}\right] .
$$

Expression (99) is the main result of this section. As a check we can take $d=1$ to recover the result 1

$$
P_{s}^{\mathrm{av}}(t) \sim \exp \left[-2 \rho \alpha_{0} \ln t\right]=t^{-2 \rho(D / r)^{1 / 2}} .
$$

Expression $(99)$ shows how the power law decay displayed in $d=1(99)$ is generalized

in arbitrary spatial dimension. The scaling form $\exp \left[-A(\ln t)^{d}\right]$ is unusual, as far as we are aware.

\subsection{Typical behaviour}

For the typical behaviour (the quenched case) in the long time limit $Q(R, t)$ is dominated by a pole at $s_{0}$ in the complex $s$ plane (49), therefore $I_{2}$, given by $(89)$, becomes

$$
I_{2} \simeq \text { Constant }-t \Gamma_{d} \int_{a}^{\infty} \mathrm{d} R R^{d-1}\left|s_{0}(R)\right|
$$

In general dimension $d \neq 1$ we were not able to perform the integral in 101 explicitly. However it is easy to show that the integral is convergent. Thus $P_{s}^{\text {typ }}(t)$ given by 87 asymptotically decays exponentially in time

$$
P_{s}^{\text {typ }}(t) \sim \exp \left[-t \Gamma_{d} \int_{a}^{\infty} \mathrm{d} R R^{d-1}\left|s_{0}(R)\right|\right] .
$$

The correction to the argument of the exponential will come from the branch point at $s=-r$ in 27) and is expected to give a subleading contribution of $O\left(t^{1 / 2}\right)$. The fact that the average and typical survival probabilities have distinct asymptotic behaviours - the average behaviour (99) decaying far more slowly than the typical behaviour (102) reflects the strong dependence on the initial conditions, noted above, whose memory is retained through resetting.

In dimension $d=1$ the integral in 101 can be evaluated in closed form. The reason is that in this case one can obtain a closed form expression for $R$ as a function of $u_{0}$ from (51)

$$
\alpha R=\alpha_{0} a-\frac{\ln \left(1-u_{0}\right)}{u_{0}^{1 / 2}} .
$$

Then one may transform the integration from $R$ to $u$ with range $0<u<1$ through $s_{0}=r(u-1)$ and letting $\epsilon \rightarrow 0$ one finds

$$
\begin{aligned}
\int_{0}^{\infty} \mathrm{d} R\left|s_{0}(R)\right| & =\frac{r}{\alpha_{0}} \int_{0}^{1} \mathrm{~d} u\left[\frac{(1-u)}{u^{3 / 2}} \ln (1-u)+\frac{1}{u^{1 / 2}}\right] \\
& =(D r)^{1 / 2} 4(1-\ln 2) .
\end{aligned}
$$

Thus in one dimension the asymptotic decay of the quenched total survival probability is exponential with explicit form [1]

$$
P_{s}^{q}(t) \sim \exp \left[-t \rho(D r)^{1 / 2} 8(1-\ln 2)\right] .
$$




\subsection{Explanation in terms of extreme value statistics}

In order to understand the asymptotic form of average survival probability (99), we consider the following simple picture. At long times the absorption probability of the target will be dominated by the searcher which started nearest to the target. Denoting the position of this searcher by $y$, the average survival probability for the many searcher problem should then be recovered by averaging the single searcher survival probability (49) over the distribution of the position $y$,

In order to obtain the distribution of the distance $y$ of the nearest searcher from the target (at the origin), we consider first the probability that a single searcher starts at distance $R>y$ from the origin

$$
\operatorname{Prob}(R>y)=\frac{\Gamma_{d}}{V} \int_{y}^{L} \mathrm{~d} R R^{d-1}=\frac{\Gamma_{d}}{d V}\left[L^{d}-y^{d}\right],
$$

where $V=\frac{\Gamma_{d}}{d}\left[L^{d}-a^{d}\right]$.

Then the probability that all $N$ searchers start at distance $R>y$ from the origin is given by, in the limit of large $N, L$ with $\rho$ fixed,

$$
\operatorname{Prob}(R>y)^{N} \simeq \exp \left\{N\left[-\left(\frac{y}{L}\right)^{d}+\left(\frac{a}{L}\right)^{d}\right]\right\} \rightarrow \exp \left[\rho \frac{\Gamma_{d}}{d}\left(-y^{d}+a^{d}\right)\right]
$$

Thus the distribution of the distance $y$ of the nearest searcher from the target is

$$
\begin{aligned}
P(y) & =-\frac{\mathrm{d}}{\mathrm{d} y} \exp \left[\rho \frac{\Gamma_{d}}{d}\left(-y^{d}+a^{d}\right)\right] \\
& =A y^{d-1} \exp \left(-\rho \frac{\Gamma_{d}}{d} y^{d}\right),
\end{aligned}
$$

where $A$ is a constant.

For a single searcher starting at $y$ the survival probability is given by 49

$$
P_{s}=\exp [-t(1-u(y))]
$$

where the function $u(y)$ is given by (51).

Thus the average survival probability is given approximately by the average of (111) with respect to 110

$$
\left\langle P_{s}\right\rangle=A \int_{0}^{\infty} \mathrm{d} y y^{d-1} \exp \left[-\frac{\rho \Gamma_{d}}{d} y^{d}-t(1-u(y))\right] .
$$

For large $t$, we expect the integral to be dominated by the saddle point $y^{*}$ of the integral with respect to $y$ which yields

$$
-\rho \Gamma_{d}\left(y^{*}\right)^{d-1}+t u^{\prime}\left(y^{*}\right)=0 .
$$

For large $t$ we expect $y^{*}$ to be large for which the asymptotic behaviour is given by (54) $u(y) \simeq 1-\epsilon^{1 / 2-\nu} \mathrm{e}^{-\alpha_{0} y^{*}(1-\epsilon)}$. The saddle point $y^{*}$ is then given by $-\rho \Gamma_{d}\left(y^{*}\right)^{d-1}+\alpha_{0}(1-$ $\epsilon) t \mathrm{e}^{-\alpha_{0} y^{*}(1-\epsilon)}=0$ which implies that asymptotically

$$
y^{*} \sim \frac{\ln t}{\alpha_{0}} .
$$


Thus at long times $t$ the survival probability is dominated by initial arrangements of searchers in which the nearest searcher is at distance $y^{*} \sim \ln t / \alpha_{0}$. The integral 112 is then dominated by

$$
\left\langle P_{s}\right\rangle \sim \exp \left[-\frac{\rho \Gamma_{d}}{d}\left(y^{*}\right)^{d}\right] \sim \exp \left[-\frac{\rho \Gamma_{d}}{d \alpha_{0}^{d}}(\ln t)^{d}\right]
$$

which recovers the asymptotic result 99 of Subsection 5.2 .

\section{Conclusion}

In this work we have studied diffusion with resetting at rate $r$ in arbitrary spatial dimension $d$. We have computed the nonequilibrium stationary state of the process given by Equation (10), which exhibits non-Gaussian behaviour. Moreover the full time-dependence is given by (5). The resetting paradigm thus presents a simple, general framework in which to study nonequilibrium properties. We have shown in this work that the spatial dimensionality produces some interesting behaviour.

In Section 3 we considered the survival probability of an absorbing target. Our central result is to compute the Laplace transform of the survival probability from which long time asymptotic behaviour may be deduced. Then in Section 4 we considered the mean time to absorption of the target. The mean time to absorption is finite and has a minimum value at an optimal resetting rate $r$.

Finally in Section 5 we consider the problem of a finite density of diffusive particles, each resetting to its own initial position. While the typical survival probability of the target at the origin decays exponentially with time regardless of spatial dimension, the average survival probability decays asymptotically as $\exp \left(-A(\ln t)^{d}\right)$ where $A$ is a constant. We have explain these findings using an interpretation as a renewal process and arguments invoking extreme value statistics.

There are many open questions concerning the resetting paradigm. In this work we have considered a single particle system under diffusion but one could generalise to other stochastic processes, for example, the Ornstein-Uhlenbeck process describing diffusion in a potential. Moreover one can generalise resetting to extended systems where the configuration of the system is reset to the initial condition at a constant rate. These dynamics generate a new class of nonequilibrium stationary states. Recent examples that have been considered include a class of reaction-diffusion systems in one dimension [24] and also growth processes described by macroscopic stochastic differential equations such

as the Kardar-Parisi-Zhang and Edwards-Wilkinson equations [25]. Naturally, studying the resetting dynamics in these extended systems in higher dimensions would be an interesting challenge.

Acknowledgements MRE would like to acknowledge funding from the EPSRC under grant EP/J007404/1. SNM acknowledges support by ANR grant 2011-BS04-013-01 WALKMAT. 


\section{References}

[1] M. R. Evans and S. N. Majumdar, Diffusion with stochastic resetting, Phys. Rev. Lett. 106, 160601 (2011).

[2] P. L. Krapivsky, S. Redner and E. Ben-Naim, A Kinetic View of Statistical Physics (Cambridge University Press, Cambridge 2010).

[3] S. C. Manrubia and D. H. Zanette, Stochastic multiplicative processes with reset events, Phys. Rev. E 59, 4945 (1999).

[4] M. Montero and J. Villarroel, Monotonous continuous-time random walks with drift and stochastic reset events, Phys. Rev. E 87, 012116 (2013)

[5] P. Visco, R. J. Allen, S. N. Majumdar, M. R. Evans, Switching and growth for microbial populations in catastrophic responsive environments, Biophysical Journal 98, 10991108 (2010)

[6] W. J. Bell, Searching behaviour: the behavioural ecology of finding resources, (Chapman and Hall, London 1991).

[7] O. Bénichou, C. Loverdo, M. Moreau, and R. Voituriez, Intermittent search strategies, Rev. Mod. Phys. 83, 81 (2011).

[8] A. Montanari and R. Zecchina, Optimizing searches via rare events, Phys. Rev. Lett. 88, 178701 (2002)

[9] G. Adam and M. Delbrück, Reduction of dimensionality in biological diffusion processes, in Structural Chemistry and Molecular Biology, A. Rich and N. Davidson Eds. (W.H. Freeman and Company, San Francisco; London, 1968).

[10] F. Bartumeus and J. Catalan, Optimal search behavior and classic foraging theory J. Phys. A: Math. Theor. 42434002 (2009)

[11] E. Gelenbe, Search in unknown environments, Phys. Rev. E 82, 061112 (2010).

[12] S. Janson and Y. Peres, Hitting times for random walks with restarts, SIAM J. Discrete Math. 26, 537 (2012)

[13] M. R. Evans and S. N. Majumdar, Diffusion with optimal resetting, J. Phys. A: Math. Theor. 44, 435001 (2011).

[14] J. Whitehouse, M. R. Evans and S. N. Majumdar, Effect of partial absorption on diffusion with resetting, Phys. Rev. E 87, 022118 (2013)

[15] M. R. Evans, S. N. Majumdar and K. Mallick, Optimal diffusive search: nonequilibrium resetting versus equilibrium dynamics, J. Phys. A: Math. Theor. 46, 185001 (2013)

[16] I.S. Gradshteyn and I.M. Ryzhik, Table of Integrals, Series and Products, Academic Press, New York (1980).

[17] A. J. Bray, S. N. Majumdar, and G. Schehr Persistence and first-passage properties in nonequilibrium systems, Advances in Physics 62, 225 (2013)

[18] S. Redner, A guide to First-Passage Processes (Cambridge University Press, Cambridge 2001).

[19] E. J. Gumbel, Statistics of Extremes (Columbia University Press, New York, 1958).

[20] M. Tachiya, Theory of diffusion-controlled reactions: formulation of the bulk reaction rate in terms of the pair probability, Radiat. Phys. Chem. 21, 167 (1983)

[21] A. Blumen, G. Zumofen, and J. Klafter, Target annihilation by random walkers, Phys. Rev. B 30, 5379 (1984).

[22] A. J. Bray and R. A. Blythe, Exact asymptotics for one-dimensional diffusion with mobile traps, Phys. Rev. Lett. 89, 150601 (2002) and references therein.

[23] R. A. Blythe and A. J. Bray, Survival probability of a diffusing particle in the presence of Poissondistributed mobile traps, Phys. Rev. E. 67, 041101 (2003)

[24] X. Durang, M. Henkel, H. Park, Statistical mechanics of the coagulation-diffusion process with a stochastic reset J. Phys. A: Math. Theor. 47, 045002 (2014)

[25] S. Gupta, S. N. Majumdar and G. Schehr, Fluctuating interfaces subject to stochastic resetting, Preprint arXiv:1312.5954 